\title{
ACTIVIDAD ANTIOXIDANTE Y ANTIENZIMÁTICA IN VITRO Y ANTINFLAMATORIA IN VIVO DEL EXTRACTO HIDROALCOHÓLICO DE Caesalpinia spinosa “TARA”
}

\author{
Activities anti-enzymatic and antioxidant in vitro and anti-inflammatory in vivo of \\ hydroalcoholic extract from Caesalpinia spinosa"tara"
}

Wilder J. Nuñez ${ }^{1}$, Raomír Quispe ${ }^{1}$, Norma J. Ramos ${ }^{1}$, Américo J. Castro ${ }^{2}$, Gloria Gordillo ${ }^{3}$

${ }^{1}$ Facultad de Farmacia y Bioquímica. ${ }^{2}$ Instituto de Investigación en Ciencias Farmacéuticas y Recursos Naturales "Juan de Dios Guevara”, ${ }^{3}$ Instituto de Investigación en Química Biológica, Microbiologíay Biotecnología” Marco Antonio Garrido Malo” Universidad Nacional Mayor de San Marcos

\section{RESUMEN}

Setuvo como objetivo explorar los potenciales antioxidante, antienzimáticoyantiinflamatorio del extracto hidroalcohólico devainas de Caesalpinia spinosa "tara". La actividad antioxidante se determinó por neutralización de los radicales 1,1-difenil-2-picril-hidrazilo (DPPH ${ }^{\bullet+}$ ) y del ácido 2,2'-azinobis (3-etilbenzotiazolin)-6-sulfónico $\left(\mathrm{ABTS}^{\bullet+}\right)$. La actividad antienzimática se evaluó por inhibición de las enzimas colagenasa y elastasa. La actividad antiinflamatoria se determinó por el método de inducción de edema plantar en ratas por $\lambda$-carragenina. Se emplearon 30 ratas albinas de cepa Holtzman, con peso promedio de $200 \pm 20$ g, distribuidas al azar en cinco grupos de seis, un control con suero fisiológico, un grupo con el estándar farmacológico indometacina y los grupos de intervención con el extracto hidroalcohólico en dosis de 50, 100 y $250 \mathrm{mg} / \mathrm{kg}$, respectivamente. El extracto mostró actividad antioxidante en los métodos $\mathrm{DPPH}\left(\mathrm{EC}_{50}=4,52 \mu \mathrm{g} / \mathrm{mL}\right)$ y $\mathrm{ABTS}^{\circ+}\left(\mathrm{EC}_{50}=14,48 \mu \mathrm{g} / \mathrm{mL}\right)$, mayor que la del patrón de referencia trolox $\mathrm{EC}_{50}=5,04 \mu \mathrm{g} / \mathrm{mL}$ y EC $50=17,04 \mu \mathrm{g} / \mathrm{mL}$, respectivamente. En la actividad antienzimática, el extracto presentó mayor potencial de inhibición de la enzima colagenasa $\left(\mathrm{IC}_{50}=196,752 \mu \mathrm{gg} / \mathrm{mL}\right) \mathrm{respecto} \mathrm{al}$ control positivo galato de epigalocatequina (EGCG) $\left(\mathrm{IC}_{50}=216,99 \mu \mathrm{g} / \mathrm{mL}\right)$, sin presentar actividad significativa para la inhibición de la enzima elastasa. En la actividad antiinflamatoria con el extracto de $250 \mathrm{mg} / \mathrm{kg}$ la inflamación disminuyó en 44,854\% a la sexta hora, sin embargo, a la misma hora la indometacina de $5 \mathrm{mg} / \mathrm{kg}$ disminuyó $48,267 \%$; los extractos de 100 y $50 \mathrm{mg} / \mathrm{g}$ también disminuyeron el edema pero en menor proporción que el estándar. A los valores obtenidos se aplicó el análisis de varianza (ANOVA) seguido de la prueba de Dunnet, con nivel de confianza del 95\% ( $p \leq 0,05)$, mostrando diferencias significativas en las medias de cada extracto en el transcurso de las horas. Se concluye que el extracto hidroalcohólico de la vainas de Caesalpinia spinosa "tara", presenta actividad antioxidante, antienzimática, antiinflamatoria.

Palabras clave: Caesalpinia spinosa “tara”, actividad antioxidante, antienzimática, antiinflamatoria.

\section{SUMMARY}

Theaim of this studiowas explore theantioxidant, anti-inflammatoryanti-enzimatic potential of hydro-alcoholic extract of Caesalpinia spinosa "tara" pods. The antioxidant activity was determined by neutralization of radical 1,1-diphenyl-2-picryl-hidrazilo (DPPH ${ }^{\bullet+}$ ) and 2,2'-azinobis acid (3-ethylbenzothiazoline)-6-sulfonic acid $\left(\mathrm{ABTS}^{\circ+}\right)$. The antienzymatic activity was assessed by inhibition of the enzymes collagenase and elastase. The antiinflammatory activity was determined by the method of induction of plantar edema in rats $\lambda$-carrageenan 30 albino rats Holtzman strain were used, with average weight $200 \pm 20$, randomly distributed in five groups of six, a control with saline, a group with indomethacin pharmacological standard and intervention groups with alcoholic extract doses of 50,100 and $250 \mathrm{mg} / \mathrm{kg}$, respectively. The extract showed antioxidant activity in $\mathrm{DPPH}\left(\mathrm{EC}_{50}=4,52 \mu \mathrm{g} / \mathrm{mL}\right)$ y $\mathrm{ABTS}^{\circ+}\left(\mathrm{EC}_{50}=14,48 \mu \mathrm{g} / \mathrm{mL}\right)$, respectively, greater than the reference pattern trolox $\mathrm{EC}_{50}=5,04 \mu \mathrm{g} / \mathrm{mL}$ and $\mathrm{EC}_{50}=17,04 \mathrm{mg} / \mathrm{mL}$. Antienzymatic activity in the extract showed greater inhibition to potential enzyme collagenase $\left(\mathrm{IC}_{50}=196,752 \mathrm{~g} / \mathrm{mL}\right)$ compared to the positive control epigallocatechin gallate $(\mathrm{EGCG})\left(\mathrm{IC} \mathrm{5}_{50}=216,99 \mathrm{mg} / \mathrm{mL}\right)$, without exhibiting significant activity for inhibiting the enzyme elastase. Antiinflammatory activity with the extract $250 \mathrm{mg} / \mathrm{kg}$ decreased inflammation $44,854 \%$ at the sixth hour, however, at the same time indomethacin $5 \mathrm{mg} / \mathrm{kg}$ decreased $48,267 \%$; extracts of 100 and 50 mg/ $\mathrm{kg}$ also reduced edema but less so than the standard. The values obtained analysis of variance (ANOVA) followed by Dunnet's test, with confidence level of $95 \%(p \leq 0,05)$ showing significant differences in the mean of each extract during the hours it wereapplied. It is concluded that the hydro-alcoholic extract of Caesalpinia spinosa"tara" pods, has antioxidant, anti-enzymatic, anti-inflammatory activity.

Keywords: Caesalpinia spinosa"Tara" antioxidant activity, anti-enzymatic, anti-inflammatory.

\section{INTRODUCCIÓN}

a "tara" es una planta originaria del Perú, empleada desde la época prehispánica en la medicina popular y en años recientes materia prima en el mercado mundial de hidrocoloides alimenticios ${ }^{(1)}$. Su contenido de metabolitos secundarios como los taninos, flavonoides y gomas hace que tenga uso medicinal y fotoprotector 
ante daños oxidativos ${ }^{(2)}$. Los antioxidantes son sustancias que hallándose presentes a bajas concentraciones respecto a las de una molécula oxidable, retardan o previene la oxidación de ese sustrato ${ }^{(3)}$. La

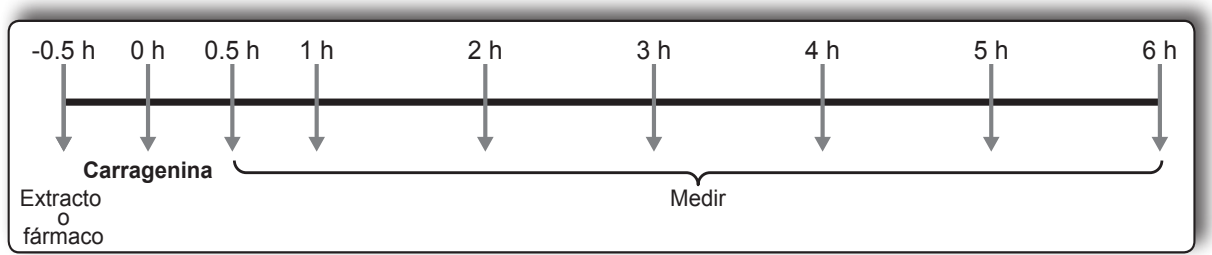

Figura 1. Esquema de administración en el edema plantar por carragenina. tara es una planta promisoria que puede actuar como antioxidante, antienzimático y antiinflamatorio ante los efectos de la radiación ultravioleta. En los últimos años se ha despertado interés por los extractos de plantas con actividades anticolagenasa y antielastasa, por la amplia variedad de constituyentes químicos que poseen, como polifenoles, flavonoides, tocoferoles, esteroles, ácidos fenólicos y taninos; que pueden actuar como poderosos bioflovonoides ${ }^{(4)}$.

La vaina es la parte más importante de la planta, ya que allí se encuentran los mayores niveles de taninos, principalmente en la cáscara. El Perú es el principal abastecedor de "tara", gracias a que posee climas y suelos que posibilitan el desarrollo de esta especie en numerosos departamentos del país. Sin embargo, pese a su importancia económica y ecológica aún no hay estudios integrales acerca de las características bioquímicas y genéticas de este recurso; ni tampoco estudios bioquímicos y genéticos relacionados con su procedencia geográfica.

El objetivo de la investigación fue determinar la actividad antioxidante antienzimática in vitro y antiinflamatorio in vivo del extracto hidroalcohólico de Caesalpinia spinosa "tara".

\section{MATERIAL Y MÉTODOS}

La investigación es experimental, prospectiva y longitudinal.

\section{Material biológico}

Las vainas de Caesalpinia spinosa "tara" fueron colectadas en la provincia de Huanta, región Ayacucho a 2628 metros de altitud y su clasificación taxonómica se realizó en el museo de Historia Natural de la Universidad Nacional Mayor de San Marcos, según el sistema de clasificación de Cronquist (1988).

\section{Obtención del extracto hidroalcohólico}

Las vainas fueron seleccionadas, limpiadas y desinfectadas con solución de cloro al $1 \%$. Posteriormente se secaron, trituraron y molieron en molino de cuchillas. El extracto hidroalcohólico se obtuvo en proceso de maceración tratando 2,o $\mathrm{Kg}$ de polvo de tara con una mezcla de etanol agua (7:3) y luego se concentró a presión reducida en un evaporador rotatorio.

\section{Determinación de la actividad antioxidante}

Se desarrolló aplicando dos métodos.

El método de captación del radical 2,2-difenil-1picrilhidrazil (DPPH) según Brand-Williams ${ }^{(5)}$, que consiste en preparar una solución stock de $40 \mathrm{mg} / 100$ $\mathrm{mL}$ de (DPPH), $1 \mathrm{mM}$ en metanol y almacenamiento a $4^{\circ} \mathrm{C}$ protegida de la luz. Se calibra el espectrofotómetro con un blanco que contiene $400 \mu \mathrm{L}$ del solvente de la muestra y $800 \mu \mathrm{L}$ de metanol, luego se coloca en un tubo de ensayo de $400 \mu \mathrm{L}$ de la muestra problema y $800 \mu \mathrm{L}$ de la solución de trabajo de DPPH y se agita. Se deja en un reposo durante 30 minutos alejado de la luz y se lee la absorbancia a $517 \mathrm{\eta m}$. Todos los extractos son medidos por triplicado a concentraciones de $0 ; 0,8 ; 1,6 ; 3,2 ; 6,4 ; 12,4 \mu \mathrm{g} / \mathrm{mL}$. Se emplea el mismo procedimiento para la sustancia patrón Trolox ${ }^{\circledast}$. Con los valores de las absorbancias obtenidas se determina el porcentaje de captación de radicales libres (DPPH), mediante la siguiente expresión:

$$
\% \text { Inhibición }=\frac{\left(A b s_{D P P H}-A b s_{\text {muestra }}\right)}{A b s_{D P P H}} \times 100
$$

El $\mathrm{IC}_{50}$ se determina a partir de la gráfica del porcentaje de inhibición y corresponde a la concentración en la que se neutraliza el 50\% de los radicales libres del DPPH.

El método de captación del radical ácido 2,2'-azinobis(3-etilbenzotiazolin)-6-sulfónico $\left(\mathrm{ABTS}^{\circ+}\right)$, se trabajó in vitro según la metodología descrita por Kuskosqui (6), el radical $\mathrm{ABTS}^{\circ+}$ se obtiene por la reacción de ABTS $7 \mathrm{mM}$ con persufato potásico $2,45 \mathrm{mM}$ mantenidos a $25^{\circ} \mathrm{C}$ y en la oscuridad durante 16 horas. Una vez formado el radical $\mathrm{ABTS}^{\bullet+}$ se diluye en agua bidestilada hasta obtener un valor de absorbancia

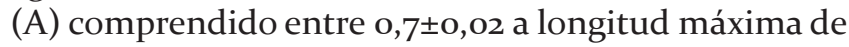
absorción $734 \mathrm{gm}$. Cada extracto se diluye con dos $\mathrm{mL}$ de etanol absoluto. A $20 \mu \mathrm{L}$ de esta solución se añaden $980 \mu \mathrm{L}$ de disolución de $\mathrm{ABTS}^{\bullet+}$ y se lee la absorbancia del blanco y del extracto a los siete minutos. Para reconocer la capacidad antioxidante de los extractos se 
elaboraron curvas estándar de Trolox ${ }^{\circledast}$. El antioxidante de referencia se ensaya a una concentración de 1,2 a 10 $\mu \mathrm{g} / \mathrm{mL}$ (concentración final) en etanol, bajo las mismas condiciones. Para las curvas a obtenerse, se calcula la regresión lineal. Con los resultados se determina el $\mathrm{IC}_{50}$ a partir de la gráfica del porcentaje de inhibición en función de la concentración, y corresponde a la concentración en la que se neutraliza el $50 \%$ de los radicales libres del $\mathrm{ABTS}^{\circ+}$. Con los valores de la absorbancia se determina el porcentaje de radicales libres $\left(\mathrm{ABTS}^{*+}\right)$ de manera semejante al método DPPH.

\section{Determinación de la actividad antienzimática}

Inhibición de enzima elastasa: el ensayo se hizo según el método descrito por Thring ${ }^{(7)}$ y consistió en incubar $25 \mu \mathrm{L}$ de muestra (a las concentraciones finales de $5,65,125,180$ y $250 \mu \mathrm{g} / \mathrm{mL}$ ) con $100 \mu \mathrm{L}$ de la enzima elastasa pancreática porcina (PE, 2,5 $\mu \mathrm{g} /$ $\mathrm{mL}$ en agua HPLC) por 15 minutos y luego agregar 125 $\mu \mathrm{L}$ del sustrato $\mathrm{N}$-succinyl-Ala-Ala-Ala-p-nitroanilide (AAAPVN, 1,6 mM en buffer THAM, Sigma 7-9 ${ }^{\circledR}, \mathrm{pH}$ $8,0)$ para que la reacción se inicie. Luego de 11 minutos se leyó la absorbancia a $410 \mathrm{jm}$ usando una lectora de placas multifunción CHAMELEON V. Se realizó un control sin muestra y se utilizó galato de EGCG como compuesto de referencia. Todos los ensayos se realizaron por triplicado.

Las $\mathrm{EC}_{50}$ (concentración efectiva media) se calcularon por análisis de regresión lineal, donde la abscisa está representada por las concentraciones de los extractos y la ordenada por el promedio de porcentaje de actividad antienzimática obtenido a partir de tres mediciones independientes.

Inhibición de enzima colagenasa: se aplicó el método descrito por Thring ${ }^{(7)}$ y consistió en incubar $100 \mu \mathrm{L}$ de muestra (a las concentraciones finales de 80, 160 y $250 \mu \mathrm{g} /$ $\mathrm{mL}$ ) con $260 \mu \mathrm{L}$ de la enzima colagenasa de Clostridium histolyticum (ChC, 1,54 unidades/mL en buffer $\mathrm{pH} 7,5$ de Tricine (N-[Tris(hidroximetil)metil]glicina) $50 \mathrm{mM}$ con $400 \mathrm{mM} \mathrm{NaCl}$ y $10 \mathrm{mM} \mathrm{CaCl}$ ) por 15 minutos y luego se agregaron $240 \mu \mathrm{L}$ del sustrato $\mathrm{N}$-[3- 2 (2-furil) acriloil]Leu-Gly-Pro-Ala (FALGPA, 2 mM en buffer $\mathrm{pH}$ 7,5 de Tricine $50 \mathrm{mM}$ con $400 \mathrm{mM} \mathrm{NaCl}$ y $10 \mathrm{mM} \mathrm{CaCl}_{2}$ ) para que la reacción se inicie. Luego de 5 minutos se leyó la absorbancia a $348 \mathrm{\eta m}$ usando un espectrofotómetro UV-Visible. Se realizó un control sin muestra y se utilizó galato de EGCG como compuesto de referencia. Todos los ensayos se realizaron por triplicado.

Las $\mathrm{EC}_{50}$ se calcularon por análisis de regresión lineal de forma similar a la elastasa.
Los porcentajes de inhibición para cada una de las dos enzimas se calculó con la siguiente fórmula:

$$
\begin{aligned}
& \text { Inhibición } \\
& \text { de la enzima }
\end{aligned}=\frac{\left(A b s^{0}-A b s^{m}\right)}{A b s^{0}}
$$

Dónde:

$A b s^{0}=$ Absorbancia del control (sin muestra)

$A b s^{m}=$ Abs. de la mezcla con sustrato - Abs. de la mezcla sin sustrato

\section{Determinación de la actividad antiinflamatoria}

Se evaluó por el método del edema de pata inducido por $\lambda$-carragenina ${ }^{(8,9)}$. Se utilizaron 30 ratas de cepa Holtzman, procedentes del Instituto Nacional Salud (INS), con peso $200 \pm 20 \mathrm{~g}$. Los animales fueron aclimatados por una semana, y distribuidos aleatoriamente en 5 grupos de 6 ratas, y tratados vía oral de la siguiente manera:

a) Grupo control: suero fisiológico (con carragenina)

b) Grupo con estándar farmacológico: indometacina (con carragenina)

c) Grupo con extractos hidroalcohólicos: 50, 100 y $250 \mathrm{mg} / \mathrm{kg}$, respectivamente (con carragenina)

Media hora después se inyectó o,1 mL de carragenina al $1 \%$ en la aponeurosis subplantar de la pata derecha. El diámetro de la pata inyectada fue determinado con pletismómetro digital a o (basal); o,5; $1 ; 2 ; 3 ; 4 ; 5$ y 6 horas después de inducir la inflamación y aplicar los tratamientos. El edema se expresa como la diferencia entre el incremento de volumen de las patas (figura 3).

La actividad antiinflamatoria se evaluó mediante el cálculo del porcentaje de inhibición del edema, según la expresión matemática:

$$
\% I=\frac{(\Delta V C-\Delta V p)}{\Delta V C}
$$

Dónde:

\%I: Porcentaje de inhibición del edema

$\Delta V C=$ Media aritmética del incremento de volumen de las patas en el grupo control ( $\lambda$-carragenina)

$\Delta V p=$ Media aritmética del incremento de volumen de las patas en el grupo problema (extractos o fármaco).

\section{RESULTADOS}

\section{Actividad antioxidante}

Los resultados obtenidos con los métodos DPPH y ATBS se muestran en las tablas 1 y 2 y en las figuras 2 y 3.

\section{Actividad antienzimática}

Los resultados del ensayo de inhibición de la enzima colagenasa se encuentran en la tabla 3, figura 4, mientras que los correspondientes a inhibición de elastasa están en la tabla 4 y figura 5. 
Se obtuvo $\mathrm{IC}_{50}$ de $216,991 \mu \mathrm{g} / \mathrm{mL}$ con el patrón de referencia galato de EGCG.

Tabla 1. Resultados de las actividad antioxidante del extracto hidroalcohólico de tara por el método de DPPH.

\begin{tabular}{cccc}
$\begin{array}{c}\text { Extracto de } \\
\text { tara ( } \mathbf{\mu g} / \mathbf{m L})\end{array}$ & $\begin{array}{c}\text { Promedio } \\
\text { absorbancia } \\
\text { a 517 } \mathbf{~ j m ~ ( * ) ~}\end{array}$ & $\begin{array}{c}\text { Desviación estándar } \\
\text { de los promedios de } \\
\text { absorbancia }\end{array}$ & $\begin{array}{c}\text { Porcentaje } \\
\text { inhibición }\end{array}$ \\
\hline 0 & $0,774(\mathrm{a})$ & 0,003 & 0 \\
0,8 & 0,492 (b) & 0,007 & 36,44 \\
1,6 & 0,472 (c) & 0,004 & 38,94 \\
3,2 & 0,433 (d) & 0,010 & 43,99 \\
6,2 & $0,358(\mathrm{e})$ & 0,014 & 53,72 \\
12,4 & 0,130 (f) & 0,002 & 83,19 \\
& & $\mathrm{EC}_{50}(\mu \mathrm{g} / \mathrm{mL})$ & 4,520 \\
\hline
\end{tabular}

*Las letras distintas en las columnas indica diferencias estadísticamente significativas usando el método de Tukey con un nivel de confianza al $95 \%$ $(p<0,05)$.

Tabla 2. Resultados de la actividad antioxidante de extracto hidroalcohólico de tara por el método de ABTS.

\begin{tabular}{cccc}
$\begin{array}{c}\text { Extracto de } \\
\text { tara ( } \mathbf{\mu g} / \mathbf{m L})\end{array}$ & $\begin{array}{c}\text { Promedio } \\
\text { absorbancia } \\
\mathbf{a} 734 \mathbf{~ j m ~ ( * )}\end{array}$ & $\begin{array}{c}\text { Desviación estándar } \\
\text { de los promedios de } \\
\text { absorbancia }\end{array}$ & $\begin{array}{c}\text { Porcentaje } \\
\text { inhibición }\end{array}$ \\
\hline 0 & 0,778 (a) & 0,010 & 0 \\
10 & 0,161 (b) & 0,007 & 39,72 \\
15 & 0,217 (c) & 0,017 & 51,8 \\
20 & 0,296 (d) & 0,012 & 61,95 \\
25 & 0,375 (e) & 0,013 & 72,11 \\
30 & 0,469 (f) & 0,033 & 79,31 \\
& & $\mathrm{EC}_{50}(\mu \mathrm{g} / \mathrm{mL})$ & 14,48 \\
\hline
\end{tabular}

* Las letras distintas en las columnas indican diferencia significativa entre las medias usando el método de Tukey con un nivel de confianza al $95 \%$ $(p<0,05)$.

Tabla 3. Resultados de la actividad de la enzima anticolagenasa del extracto hidroalcohólico de tara.

\begin{tabular}{cccc}
$\begin{array}{c}\text { Extracto de } \\
\text { tara ( } \mathbf{\mu g} / \mathbf{m L})\end{array}$ & $\begin{array}{c}\text { Promedio } \\
\text { absorbancia } \\
\mathbf{a} \mathbf{3 4 8} \mathbf{\text { jm }} \text { (*) }\end{array}$ & $\begin{array}{c}\text { Desviación estándar } \\
\text { de los promedios de } \\
\text { absorbancia }\end{array}$ & $\begin{array}{c}\text { Porcentaje } \\
\text { inhibición }\end{array}$ \\
\hline 0 & 0,837 (a) & 0,0187 & 0 \\
100 & 0,555 (b) & 0,0098 & 33,63 \\
200 & 0,370 (c) & 0,0130 & 55,78 \\
400 & 0,208 (d) & 0,0095 & 75,14 \\
& & $\mathrm{IC}_{50}(\mu \mathrm{g} / \mathrm{mL})$ & 196,752 \\
\hline
\end{tabular}

* $L$ as letras distintas en las columnas indican diferencia significativa usando en método de Tukey con un nivel de confianza al $95 \%(p<0,05)$.

Tabla 4. Resultados de la actividad de la enzima elastasa del extracto de tara.

\begin{tabular}{|c|c|c|c|}
\hline $\begin{array}{c}\text { Extracto de } \\
\operatorname{tara}(\mu \mathrm{g} / \mathrm{mL})\end{array}$ & $\begin{array}{l}\text { Promedio } \\
\text { absorbancia } \\
\text { a } 410 \text { jm (*) }\end{array}$ & $\begin{array}{l}\text { Desviación estándar } \\
\text { de los promedios de } \\
\text { absorbancia }\end{array}$ & $\begin{array}{l}\text { Porcentaje } \\
\text { inhibición }\end{array}$ \\
\hline 0 & $0,144(a)$ & 0,0045 & 0 \\
\hline 50 & 0,137 (b) & 0,0017 & 4,640 \\
\hline 125 & 0,107 (c) & 0,0247 & 25,754 \\
\hline 180 & 0,095 (d) & 0,0151 & 33,643 \\
\hline \multirow[t]{2}{*}{250} & $0,078(\mathrm{e})$ & 0,0100 & 34,339 \\
\hline & & $\mathrm{IC}_{50}(\mu \mathrm{g} / \mathrm{mL})$ & 348,396 \\
\hline
\end{tabular}

*Las letras distintas en las columnas indican diferencia significativa entre las medias usando el método de Tukey con un nivel de confianza al $95 \%$ $(p<0,05)$.

\section{Actividad antiinflamatoria}

El análisis estadístico para los resultados son expresados en promedios (o medias) para todas las mediciones obtenidas. Se realizó mediante el análisis de varianza (ANOVA) seguido de una prueba de Dunnett y la prueba de Grubbs para la determinación de valores atípicos, con un nivel de confianza de $95 \%(p<0,05)$ empleando el programa Minitab (17).

\section{DISCUSIÓN}

Las vainas secas de Tara son conocidas en la medicina tradicional por aliviar problemas inflamatorios de garganta, ojos, sinusitis, infecciones vaginales y micóticas, heridas crónicas, reumatismo, etc. En ratas, se ha descrito que durante las primeras tres horas después de la administración por vía plantar, produce un comportamiento bifásico; la primera fase es mediada por la liberación de

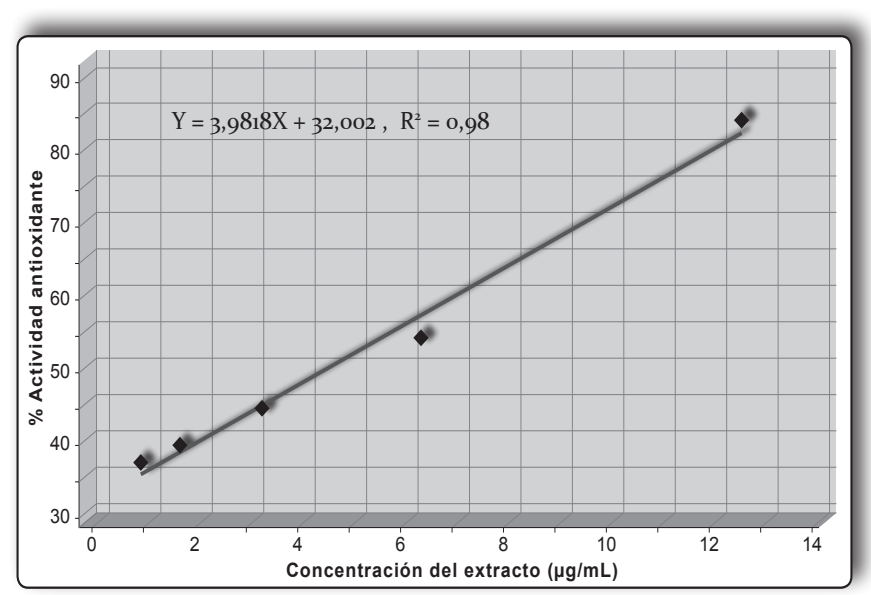

Figura 2. Correlación entre extractos hidroalcohólico de la vaina de tara vs \% de inhibición del radical DPPH.

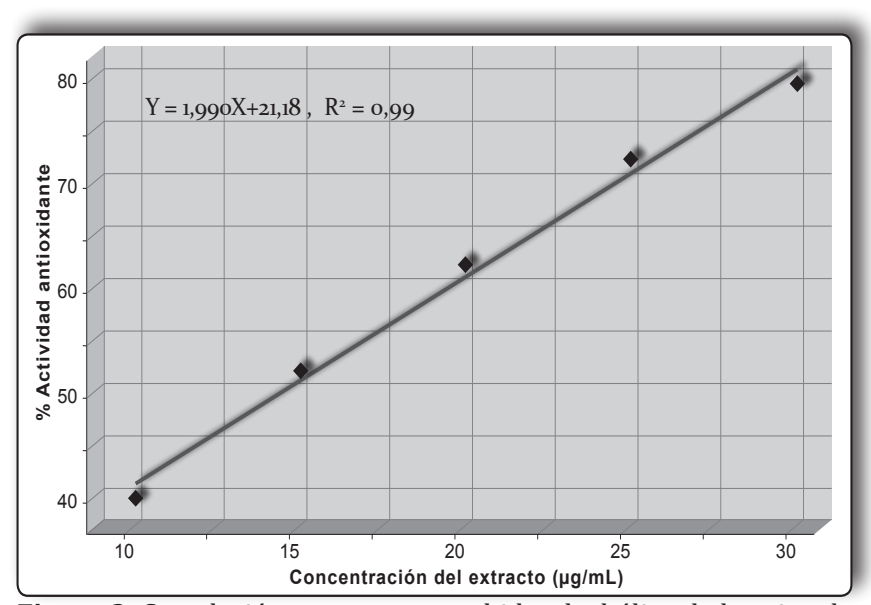

Figura 3. Correlación entre extractos hidroalcohólico de la vaina de tara vs \% de inhibición del radical ABTS *. 


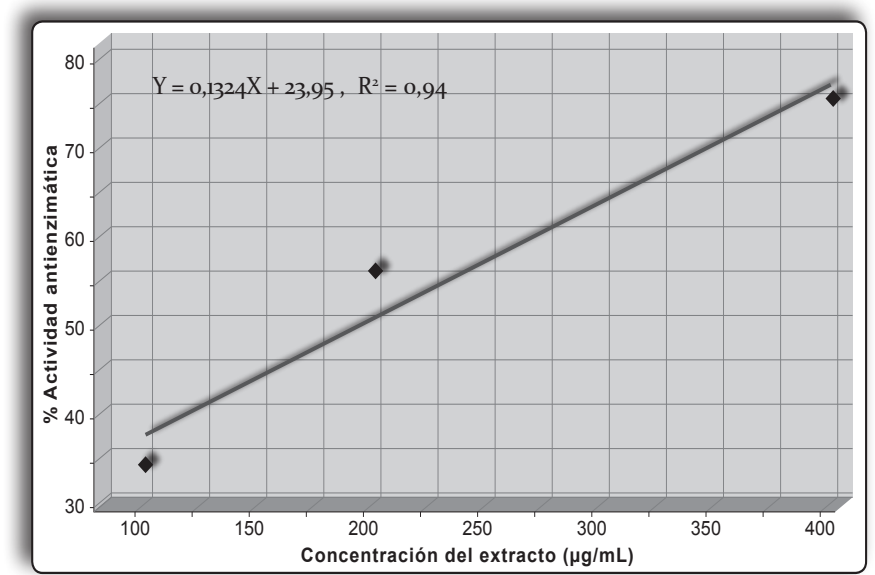

Figura 4. Correlación entre extractos hidroalcohólicos de la vaina de tara vs \% de inhibición de la enzima colagenasa.

histamina y serotonina, mientras que en la segunda se liberan prostaglandinas, bradiquinina, proteasa y lisosoma, con un efecto máximo alrededor de tres horas ${ }^{(10)}$.

En la figura 6 se observa el aumento del volumen de la pata inflamada siendo notorio el efecto de la carragenina. En en la sexta hora disminuyó la curva de carragenina, posiblemente por la acción antiinflamatoria del propio organismo. Los resultados indican que la acción del extracto con mayor concentración es comparable con indometacina a la quinta hora.

No se evidenciaron diferencias significativas entre los extractos y el fármaco de referencia en los primeros 30 y 60 minutos de aplicación, en cuanto a la actividad antiinflamatoria $(\mathrm{p}=0,78$ y $\mathrm{p}=129$, respectivamente, método Dunnett). A partir de la segunda hora, los extractos empezaron a diferenciarse del patrón, observándose a la sexta el pico más alto de inhibición de la indometacina y el extracto 250 $\mathrm{mg} / \mathrm{kg} \quad(48,267 \%$ y $44,854 \%$ de disminución de la inflamación, respectivamente).

En la figura 6, se evidencia que a mayor concentración del extracto hay mayor eficiencia antiinflamatoria. La disminución del edema se debe

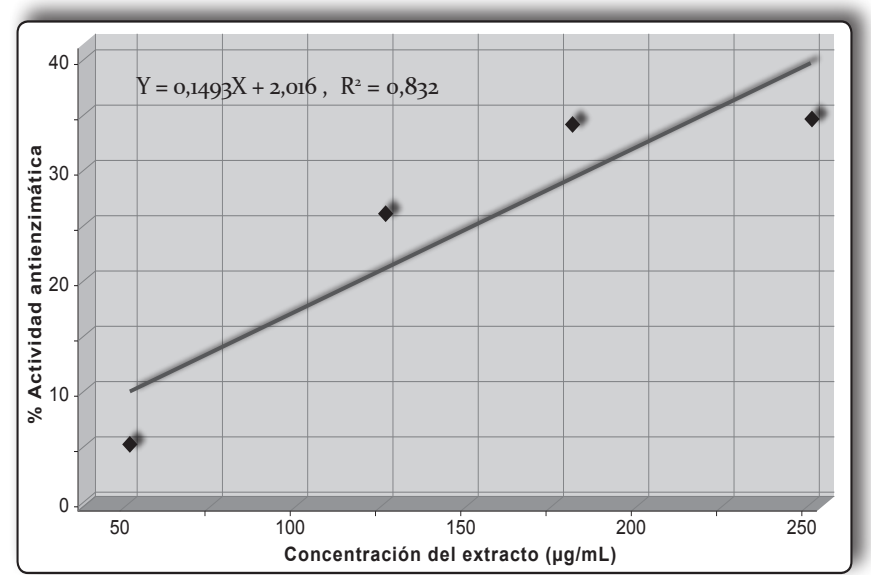

Figura 5. Correlación entre extractos hidroalcohólico de la vaina de tara vs \% de inhibición de la enzima elastasa.
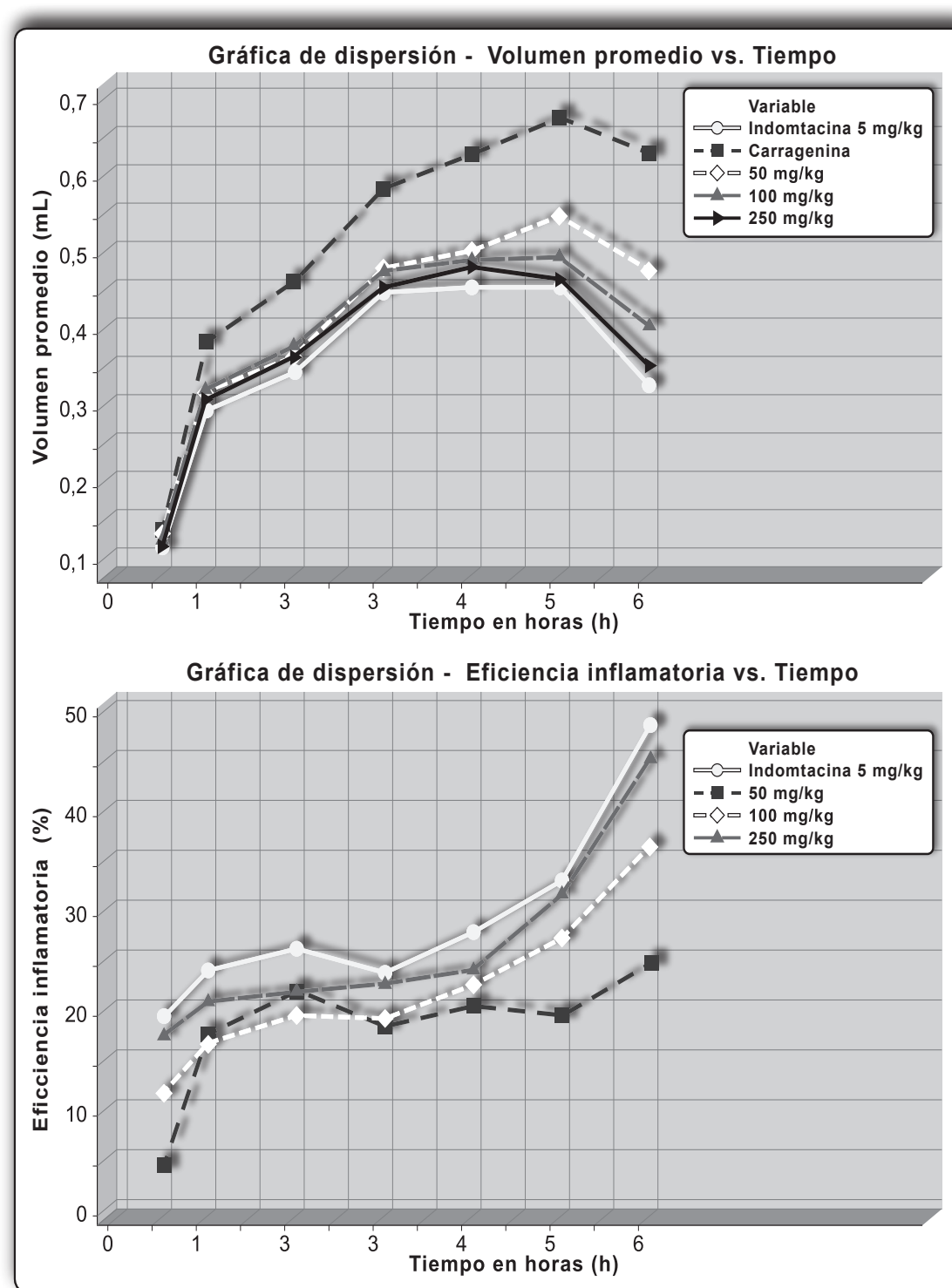

Figura 6. Volumen promedio de la pata inflamada y eficiencia inflamatoria. 
Tabla 5. Promedio del volumen de la pata inflamada en las distintas horas.

\begin{tabular}{|c|c|c|c|c|c|c|c|}
\hline \multirow{2}{*}{ Sustancias } & \multicolumn{7}{|c|}{ Volumen promedio de pata inflamada (mL) } \\
\hline & 0.5 hora & 1 hora & 2 horas & 3 horas & 4 horas & 5 horas & 6 horas \\
\hline Carragenina & $0,1342^{\mathrm{A}}$ & $0,3787^{\mathrm{A}}$ & $0,4583^{\mathrm{A}}$ & $0,5785^{A}$ & $0,6242^{\mathrm{A}}$ & $0,670^{\mathrm{A}}$ & $0,625^{\mathrm{A}}$ \\
\hline Extracto $50 \mathrm{mg} / \mathrm{kg}$ & $0,1287^{\mathrm{A}}$ & $0,3134^{\mathrm{A}}$ & $0,3600^{\mathrm{A}}$ & $0,4731^{\mathrm{A}}$ & 0,4976 & 0,5425 & 0,4717 \\
\hline Extracto $100 \mathrm{mg} / \mathrm{kg}$ & $0,1192^{\mathrm{A}}$ & $0,3168^{A}$ & 0,3717 & $0,4700^{\mathrm{A}}$ & 0,4850 & 0,4904 & 0,4000 \\
\hline Extracto $250 \mathrm{mg} / \mathrm{kg}$ & $0,1111^{\mathrm{A}}$ & $0,3017^{\mathrm{A}}$ & 0,3600 & 0,4500 & 0,4774 & 0,4609 & 0,3447 \\
\hline Indometacina $5 \mathrm{mg} / \mathrm{kg}$ & $0,1085^{\mathrm{A}}$ & 0,2891 & 0,3400 & 0,4433 & 0,4518 & 0,4508 & 0,3233 \\
\hline ANOVA & $\mathrm{p}=0,781$ & $\mathrm{p}=0,129$ & $\mathrm{p}=0,027$ & $\mathrm{p}=0,026$ & $\mathrm{p}=0,001$ & $\mathrm{p}=0,000$ & $\mathrm{p}=0,000$ \\
\hline
\end{tabular}

${ }^{*} \mathrm{p}<0.05$ existe diferencias significativas (ANOVA).

*Las medias no etiquetadas con la letra A son significativamente diferentes de la media del nivel de control "carragenina" (DUNNETT).

a la acción de grupos fenólicos, relacionados con la inhibición de diversas enzimas implicadas en el metabolismo del ácido araquidónico -ciclooxigenasa, lipoxigenasa, NADPH oxidasa y xantina oxidasa-, que reducen el estrés oxidativo y a flavonoides (11). In vitro, los flavonoides polihidroxilados actúan preferentemente por la vía 5-lipooxigenasa, mientras quelos menos hidroxilados inhiben fundamentalmente la vía ciclooxigenasa. In vivo, sin embargo, parecen comportarse como inhibidores duales (12). Esta diferentcia de comportamiento, no exclusiva de flavonoides, se debe a la biotransformación que sufren en el organismo.

La indometacina $5 \mathrm{mg} / \mathrm{kg}$ es un AINE que actúa sobre la cascada del ácido araquidónico inhibiendo no selectivamente a la COX, que suprime la formación de prostaglandinas y también inhibe la marginación, adhesión y migración de leucocitos, por lo que se considera un eficaz agente antiinflamatorio.

Como la vaina de tara presenta alto porcentaje de compuestos fenólicos -comprobado in vitro por diversos autores-, en el presente estudio se esperaba mayor actividad antiinflamatoria ya que esta propiedad es atribuíble a la biodisponibilidad de estos grupos. Sin embargo, los polifenoles, en mayor porcentaje, no siempre son los más activos en el organismo, ya sea por su menor actividad intrínseca, baja absorción intestinal, que son altamente metabolizados o de excreción rápida; en general su metabolismo se produce a través de una secuencia de reacciones similares a la decodificación metabólica que sufren muchos xenobióticos.

Para evaluar la actividad antioxidante se utilizaron los métodos in vitro ABTS y DPPH, que proporcionan una idea aproximada de lo que ocurre en situaciones complejas in vivo ${ }^{(13)}$.

El DPPH reportó un $\mathrm{EC}_{50}$ de $4,520 \mu \mathrm{g} / \mathrm{mL}$ para el extracto hidroalcohólico de tara y 5,04 $\mu \mathrm{g} / \mathrm{mL}$ para la solución patrón trolox. El ensayo con ABTS presentó un $\mathrm{EC}_{50}$ de $14,48 \mu \mathrm{g} / \mathrm{ml}$ para el extracto hidroalcohólico y $17,04 \mu \mathrm{g} / \mathrm{mL}$ para la solución patrón trolox. En las tablas 1 y 2 se puede observar que el extracto hidroalcohólico de tara posee buena actividad antioxidante en los ensayos DPPH y ABTS, siendo inclusive mayor a la del control positivo.

Se demostró que todos los valores obtenidos en ambas pruebas, según el análisis estadístico de ANOVA, presentan un $p<0,05$, por lo que se acepta que las diferentes concentraciones del extracto hidroalcohólico de tara poseen estadísticamente diferencias significativas entre sí.

Los flavonoides son antioxidantes, estando sus propiedades anti-radicales libres dirigidas fundamentalmente a los radicales hidróxidos y superóxidos, especies altamente reactivas implicadas en el inicio de la cadena de peroxidación lipídica.

Existen diferencias entre los resultados aquí obtenidos y los de otros investigadores. Esto podría deberse a diversos factores que influyen en su producción y productividad, ya que se ha descrito que la capacidad antioxidante en un alimento vegetal no proviene sólo de la suma de las capacidades antioxidantes de sus componentes, sino que el microambiente en que se halla, influiría sobre dichos componentes para que interactúen entre sí produciendo efectos sinérgicos ${ }^{(14)}$. Sin embargo, en la tara hay que considerar la presencia de metabolitos antioxidantes ${ }^{(15)}$, como fenoles y flavonoides, cuyo contenido puede ser influido por la altitud y la radiación de la región de origen.

El 8o\% del peso seco de la piel está constituido por colágeno, que es responsable de su textura; de otro lado, la elastina proporciona elasticidad a arterias, pulmones, ligamentos y, por supuesto, la piel. La colagenasa y elastasa son enzimas que 
degradan, respectivamente, ambas proteínas causando la aparición de arrugas, y la tara podría tener características inhibitorias para las dos.

La actividad anticolagenasa del extracto de tara aparece desde las concentraciones bajas y se incrementa en las altas. La prueba usada posee concentraciones constantes de enzima y sustrato, aunque se incremente la concentración de la muestra, habrá un límite que se expresa en la absorbancia. En la tabla 3 se muestran valores de inhibición que oscilan en el rango de 33,63 a $75,14 \%$, con $\mathrm{IC}_{50} 196,752 \mu \mathrm{g} / \mathrm{mL}$ para el extracto de tara, que comparado con el $\mathrm{IC}_{50} 216,991 \mu \mathrm{g} / \mathrm{mL}$ del compuesto de referencia demuestran que el extracto posee mayor actividad anticolagenasa que el EGCG. Según el análisis estadístico ANOVA presentan un $p<0,05$, por lo que las medias de las absorbancia son estadísticamente diferentes en cada concentración del extracto.

La capacidad de inhibir esta enzima se debe a la presencia de taninos condensados, los cuales tienen actividad quelante, formando precipitados con iones metálicos, como el zinc, metal componente del centro activo de la colagenasa, que impide la unión de ésta con el sustrato; por tanto, un mayor contenido de taninos podría asociarse con un incremento en la fuerza de interacción de sus componentes con la colagenasa, que a su vez podría relacionarse con su actividad inhibitoria (16). Es un tipo de inhibición reversible competitiva.

Respecto a los resultados, el potencial antielastasa (tabla 4) se obtuvo un $\mathrm{IC}_{50}$ de 348,396 $\mu \mathrm{g} / \mathrm{mL}$ para los extractos de tara, en comparación de $12,1266 \mu \mathrm{g} / \mathrm{mL}$ para el compuesto de referencia EGCG. La figura 3 muestra la creciente inhibición de las enzimas en presencia del extracto, que posiblemente se debió a la presencia de flavonoides libres, glucósidos y taninos condensados. Se observó que no presenta actividad antielastasa considerable.

Los valores de la actividad antiinflamatoria, antioxidante y la capacidad enzimática, fueron sometidos al análisis de varianza (ANOVA) con un nivel de significancia del 95\% ( $\mathrm{p}<0,05)$.

\section{CONCLUSIONES}

El extracto hidroalcoholico de Caesalpinia spinosa tara mostró actividad antioxidante in vitro (DPPH y ABTS) mayor que el estándar de referencia Trolox, asimismo inhibe la enzima colagensa con mayor potencia que el control positivo EGCG, aunque no inhibe la elastasa.

La planta posee antiinflamatoria dosis dependientes con diferencias significativas entre las medias de las sustancias entre la segunda y sexta horas, sin embargo, esta no es siginificativa al compararla con el patrón de referencia (indometacina) a las mismas concentraciones.

\section{REFERENCIAS BIBLIOGRÁFICAS}

1. De la Cruz P. Aprovechamiento integral y racional de la tara Caesalpinia spinosa - Caesalpinia tinctoria. Revista de Instituto de Investigación FIGMMG. 2004; 7(14): 64-73.

2. Tomás-LorenteF. Aportación al estudiodelos flavonoides. Madrid: Ed. Real Academia de Doctores de España; 2004.

3. Garcia BL, Vicente GG, Rojo DD, Sánchez GE. Plantas con propiedades antioxidantes. Rev Cubana Invest Biomed, 2001; 20(3): 231-35.

4. Kim Y, Uyama H, Kobayashi S. Inhibition effects of $(+)$-catechinae-dehide polyandensates on proteinases causigproteolytic degradation of extracellular matrix. Biochem Biophys Res Commun. 2004; 320: 256-61.

5. Brand-Williams W, Cuvelier ME, Berset C. Use of radical method to evaluate antioxidant activity. Food Science And Technology. 1995; 25(1): 25-30.

6. Kuskoski EM, Asuero AG, Troncoso AM, ManciniFilho J, Fett R. Aplicación de diversos métodos químicos para determinar actividad antioxidante en pulpa de frutos. Cienc Tecnol Aliment. 2005; 25(4): 726-32.

7. Thring, TS, Hili P, Naughton DP. Anti-Collagenase, antielastase and anti-oxidant activities of 21 plants. BMC Complement Altern Med. 2009; 9:t 27.

8. Sughisita E, Amagaya S, Ogihara Y. Antiinflammatory testing methods: Compararive evaluation of mice and rats. J Pharmacobiodyn. 1981; 4(8): 565-75.

9. Winter CA, Porter CA. Effect of alteration in side chain uppon antiinflamatory and liver glycogens activities of hidrocortisone esters. J Am Pharm Assoc. 1957; 46(9): 509-15.

10. Chakraborty A, Devi R, Rita S, Sharatchandra K, Singh T. Preliminary studies on antiinflammatory and analgesic activities of Spilanthes acmella in experimental animal models. Indian J Pharmacol. 2004; 36(3): 148-50.

11. Shigeoka S, Ishikawa T, Tamoi M. Regulation and function of ascorbate peroxidase isoenzymes. J Exp Bot. 2002; 53(372): 1305-19.

12. Ferrándiz L, Alcaraz M. Anti-inflammatory activity and inhibition of arachidonic acid metabolism by flavonoids. Agents Actions. 1991; 32: 283-8. 
13. Castañeda C, Ramos Ll, Ibañez V. Evaluación de la capacidad antioxidante de siete plantas medicinales peruanas. Revista Horizonte Médico. 20o8; 8(1): 5672.

14. Muñoz J, Ramos E, Alvarado-Ortiz U, Castañeda C. Evaluación de la capacidad antioxidante y contenido de compuestos fenólicos en recursos vegetales promisorios. Rev Soc Quím Perú. 2007; 73(3): 142-19.

15. Fukumoto L, Mazza G. Assessing antioxidant and prooxidant activities of phenolic compounds. Journal of Agricultural and Food Chemistry. 2000; 48: 3597-3604.
16. Melzig MF, Loser B, Ciesielski S. Inhibition of neutrophil elastase activity by phenolic compounds from plants. Pharmazie. 2001; 56: 967-70.

Manuscrito recibido el: 15/02/2016

Aceptado para su publicación el: 20/04/2016

\section{Correspondencia}

Nombre: $\quad$ Wilder Javier Núñez Enero

Dirección: MzG1 Lt.25 Urb. SantoDomingo-Carabayllo e-mail: 